\title{
ON THE ENERGY EFFICIENCY OF ELECTROMECHANICAL SYSTEMS WITH AN ELECTROMAGNETIC VIBRATION EXCITER
}

\author{
Nuraliev A. K. ${ }^{1}$, Amirov T. ${ }^{2}$, Esenbekov A.J. ${ }^{3}$ \\ ${ }^{1}$ Tashkent State Technical University named after Islam Karimov (Tashkent, Republic of Uzbekistan) \\ ${ }^{2}$ Tashkent State Technical University named after Islam Karimov (Tashkent, Republic of Uzbekistan) \\ ${ }^{3}$ Karakalpak State University named after Berdakh (Nukus, Republic of Karakalpakstan)
}

\begin{abstract}
Study shake the phenomena in the electromechanical systems (EMS) are presented by permanent interest for the wide circle of researchers. The most effective source of vibrations in many cases is an electromagnetic causative vibration exciters (EMCV) in that there is transformation of electric power to energy of mechanical vibrations, that is related to the change of inductance. Electromagnetic vibration exciters have a number of significant advantages over other types of exciters. Due to the absence of rubbing parts they have high reliability and durability. They allow you to smoothly adjust the amplitude of vibrations over a wide range. EMCV are easy to integrate into production lines, they have easy maintenance, low operating costs and extensive automation capabilities. This phenomenon presents interest at development of electromechanics devices for intensive vibrotechnologies. Especially development guided on frequency of resonant EMCV promotes the economy of consumption of electric power and productivity of vibrotechnique. To the article the results of theoretical and experimental researches are driven on the increase of energy efficiency of EMCV and determination of output-input ratio. It is shown that losses of electric power in EMS with EMCV related to the origin of permanent constituents and ultra-harmonics of current, choice of magnetic chain and dynamic.
\end{abstract}

\section{Introduction}

As a result of theoretical analysis and experimental research, the main mechanical characteristics of EMCV and their relationship with energy indicators were identified, which indicates the ways of creating more advanced energy-efficient devices [1-11].

The results of the study make it possible to determine the energy efficiency of the EMCV and can be useful in the design and construction of such devices.

Theoretical and experimental studies show that the change in inductance in the magnetic circuit has a significant effect on the energy of oscillations in EMCV [2,3].

Since the flux linkage in the vibration exciter winding with unsaturation of the magnetic system is equal to:

$$
\psi=L(x) \cdot i(\omega t)
$$

then the shape of the $\mathrm{L}(\mathrm{x})$ curve has a decisive influence on the power value. It is determined by the configuration of the magnetic circuit and the ratio of the magnetic conductivities for the working magnetic flux and leakage flux at different positions of the armature. Consequently, the power of the EMEV will largely be determined by the coefficient of stiffness of the springs with and the mass ma and $\mathrm{m} \rho$. In this case, the flux linkage of the EMCV winding in the generating approximation with forced oscillations will change with the frequency of the source $\omega_{B}$ :

$$
\begin{array}{r}
\psi_{0}=I_{0} L_{0} \sin \left(\omega_{B} t-\varphi_{0}\right) \\
\text { where } I_{0}=U_{m}^{m}\left[\sqrt{R^{2}+\left(\omega_{B} L_{0}\right)^{2}}\right], \\
\varphi_{0}=\operatorname{arctg} \frac{\omega L_{0}}{R}, \omega_{B}=\sqrt{\frac{c}{m}}=\sqrt{c \frac{m_{a}+m_{p}}{m_{a} m_{p}}}
\end{array}
$$

then the current will have the form, given $\psi=L i$

$$
i=I_{0} \exp \left[m \cos \left(\omega_{B} t-\varphi\right)-2 m_{\rho} \cos ^{2}\left(\omega_{B} t-\varphi\right)\right] \sin \left(\omega_{B} t-\varphi_{0}\right)
$$


According to equations $(2,3)$ in Fig. 1 the dependencies $\psi(i)$ and $i(t)$. The area bounded by the curve $\psi(i)$ is proportional to the mechanical work of the EMCV [3]. The power of the EMCV can then be determined as follows:

$$
P=\frac{1}{T} \int_{0}^{T} i d \psi
$$

Let us expand the amplitude factor in equation (3) in a power series and, substituting the resulting expression for the current into integral (4), we obtain

$$
P=\omega_{B} \frac{L_{0} I_{0}^{2}}{2} M_{1} \cos \theta
$$

From equation (5) it follows that the power of the EMCV increases with an increase in the relative change in inductance and depends on the degree of concavity of the curve $\mathrm{L}(\mathrm{x})$. Coefficient $\mathrm{M}_{1}$ characterizes the efficiency of energy conversion. The relationship of this coefficient with the electrical parameters of the EMCV and with the required maximum force acting on the armature can be obtained as follows:

$x=A \sin \left(\omega_{B} t-\varphi_{0}-\theta\right)$ - moving anchor

$F=F_{m} \cos \left(\omega_{B} t-\varphi_{0}\right)-$ the force acting on the armature with the working body, then the mechanical power of the EMCV is equal to:

$$
P=\frac{1}{T} \int_{0}^{T} F d x=\omega A F_{m} \cos \theta
$$

Equating the right-hand sides of equations $(5,7)$, we obtain:

$$
F_{m}=\frac{M_{1}}{A} \frac{L_{0} I_{0}^{2}}{2}
$$

In the case of power supply from the inverter in singlegap mode, considering that

$\Phi_{0}=\lambda-\cos \tau$

where $\quad \Phi_{0}=\frac{\pi \psi_{0} \omega_{B}}{4 v_{0} m_{0}} ; v_{0}=\sqrt{\frac{c}{m \omega_{6}^{2}}}$

$0 \leq \tau \leq 2 \pi$

you can get the power consumed by EMVV (average for the period)

$$
P=\frac{16 U_{0}^{2} m_{0}\left(M_{1} \cos \varphi_{1}+M_{2} \cos 2 \varphi_{1}\right)}{\pi^{2} \omega L_{0}}
$$

where

$$
\left\{\begin{array}{l}
M_{1}=\frac{1}{2}\left[m\left(\lambda-\frac{3}{2} m_{\rho} \lambda\right)+\frac{m}{8}\left(m^{2} \lambda-5 m_{\rho}^{2}\right)+\ldots\right] \\
M_{2}=\frac{1}{4}\left[m_{\rho}\left(1-m_{\rho}\right)+\frac{m^{2}}{2}\left(m_{\rho}-\frac{1}{2}\right)+\frac{5}{8} m_{\rho}^{3}-\ldots\right]
\end{array}\right.
$$

When tuned to resonance in frequency $\omega \mathrm{B}$, the component $M_{2} \cos 2 \varphi_{1} \rightarrow 0$ and the power is approximately equal to

$$
P \approx \frac{16 U_{0}^{2} m_{0}^{2}}{\pi^{2} \omega L_{0}} M_{1} \cos \varphi_{1}
$$

As you can see, the nonlinear nature of the change in inductance from displacement $\mathrm{x}$ affects the magnitude of the power of the EME through the conversion factor $\mathrm{M}_{1}$ and allows you to determine the rational form of the magnetic circuit when designing the EMCV.

Practically important data in such systems is energy efficiency. Consider the balance of power energies in systems powered by an alternating current network:

$$
P=P_{э м}+\Delta P_{n p}+\Delta P_{c m}+\Delta P_{M}+\Delta P_{b}+\Delta P_{b c n}
$$

where: $P_{э М}-$ power consumption of EMCV;

$\Delta P_{n p}=(0,01-0,05) P-$ power loss in the rectifier; $\Delta P_{c m}-$ loss in the steel core of the vibration exciter; $\Delta P_{M}-$ loss in copper winding;

$\Delta P_{b}-$ loss of armature power;

$\Delta P_{b c n}-$ loss in auxiliary equipment (in control systems).

Determination of the armature power loss $\Delta \mathrm{P}_{\mathrm{b}}$ is one of the most difficult tasks of vibration equipment. Their number depends on transportation conditions, vibrations, pressure characteristics of materials, etc.

Currently, there is no practically suitable methodology for determining them experimentally. 
The average value of the useful power $\Delta \mathrm{P}_{\mathrm{b}}$, required to maintain steady vibration is:

$$
\Delta P_{b}=\frac{1}{T} \int_{0}^{T} Q * X * \omega \cos \alpha d t
$$

where, $\alpha$ - angle between power vector $Q$ and vibration transmission $\mathrm{X}$ with load sides. Higher harmonics create dynamic pressure on the structure of the electromagnetic vibration exciter. Does not allow for significant performance gains. This is why the energy to excite higher frequencies can be defined as loss. Average of these losses, $\Delta P_{b}$, can be determined from [L12].

The active net power of the network is:

$$
P=U I \cos v
$$

voltage

where $U$ - effective value of the mains

I - operating effective value of the first harmonic of the current.

$v$ - switching angle of the thyristor converter.

The efficiency of energy conversions in the control system of an electromagnetic vibration exciter can be represented as follows:

$$
\eta=\frac{P_{\ni M}}{P_{э м}+\Delta P_{n p}+\Delta P_{M}+\Delta P_{c m}+\Delta P_{b c n}+\Delta P_{b}}
$$

The other most important energy data is the power factor $\lambda[3]$.

$$
\lambda=\frac{P}{S}
$$

Coefficient $\lambda$ is determined at the input of one-way bridge receivers supplying the converter and displacing the winding according to known formulas. In this case:

$$
\lambda=\frac{2 \sqrt{2}}{\pi} \cos v
$$

\section{Conclusion}

1. The power of the EME is largely determined by the rational choice of the magnetic circuit and can be adjusted using the energy conversion factor.

2. The power of the vibration exciter increases with an increase in the relative change in inductance and depends on the degree of concavity of the curve $\mathrm{L}$ (x).

3. Increasing the energy efficiency of the exciter can be achieved by reducing the power using the fundamental harmonic without loss. Working on the fundamental harmonic to attenuate the second higher harmonic, it is necessary to increase the constant components of the magnetic flux, the amplitudes of the first and the lowest harmonics.

4. 4The above equations make it possible to determine the power losses and the efficiency of the EMCV and can be useful in the design of electromechanical devices.
Capacitance factor boosting can be enabled for the rectifier input to the capacitor bank or using a dedicated rectifier circuit such as a tunnel diode.

Increasing energy efficiency can be done by reducing power using lossless harmonics. In systems with a constant amplitude offset, the transmission oscillation usually carries a constant component, the first, second and third harmonics.

Working on the first harmonic to attenuate the second harmonic, it is necessary to increase the constant component of the magnetic flux, the amplitudes of the first and lowest harmonics. That is why the quality of the constant bias should be chosen at 1,1 - 1,3 times the amplitude of the constant maintenance of the total flow. Another method of increasing efficiency is generally accepted.

In some cases, the maximum average power has been practically reached, and therefore compliance with the specified criteria becomes necessary and sufficient. In the linear subsystem, the average power reaches:

$$
P_{b \max }=\frac{F_{a}^{2} \omega}{4\left(c-m \omega^{2}\right)}=\frac{F_{a}^{2} \omega}{4 m\left(\omega_{0}^{2}-\omega^{2}\right)}
$$

where $F_{a}$ - forced force amplitude;

$\omega$ - resonant frequency;

$\mathrm{c}$ - elastic spring system;

$\mathrm{m}$ - the mass of the waste part is connected together with the mass of pressure;

$\omega_{0}$ - natural frequency of the mechanical subsystem. Experimental studies show that the constant current component and higher harmonics cause additional losses in the electromagnet winding and require an overestimation of the EMCV power.

\section{Literature}

1. Ilyin M. M. Theory of oscillations. Text book for Universities M. M. Ilyin, K. S. Kolesnikov, Yu. S. Saratov; Edited byK. S. Kolesnikov, $2^{\text {nd }}$ edition MSTU named after N. E. Bauman, 2003 - page № 272

2. Hoshimov, F.A., Bakhadirov, I.I., Erejepov, M., Djumamuratov, B. (2019) Development of method for normalizing electricity consumption E3S Web Conf 139 doi:10.1051/e3sconf/201913901074

3 . Bessonov L. A. Nonlinear electrical circuits. $5^{\text {th }}$ edition. Moscow. Graduate school 2015, стр. 375

4. Ibadullaev M.I., Nuraliev A.K., Esenbekov A.J., Nazarov A.I. Resonant electromagnetic vibration exciter with feedback. 2020. Vestnik, MEI, №1. pages № 62-66

5. NazarovA. I., IbadullaevM. I., NuralievA. K. On vibrational electro mechanical systems with an electromagnetic electric exciter. 
Magazine: Problems of energy and resource saving. Tashkent № 3-4, 2018, pages № 158-161

6. S. H. Chorodhury, M. M. Tillahojaev. Electro-Magnetic Vibro-Exciter of Oscillaritions controlled by Phase Feedback. Journal of the Institutions of engineers Bangladesh. vol. 22 №2 april 2004, p. 67-71.

7. Korablev S.S. About selfoscillations of an Electromechanical exciter. Proceedings Of The Universities. Elecromechanics 1963 №6 pages№ 723-728.

8. AntipovV.I., EfremenkovE.E., RuinA.A., SubbotinYu.O. Increasing the energy efficiency of vibration mechanisms due to the excitation of a low-frequency resonant mode of vibrations.Glass and ceramics 2007 №5 page№ 13-16.
9. AfanasievA.I., ZakamennykhYu.G. Analysis of resonant vibro transport vehicles.Proceedings of The Universities. Mining journal №8 2008 pages 101-106.

10. M. Ibadullaev, A. Nuraliev, A. Esenbekov. Research of an electromagnetic vibrator with a non-linear power supply. IOP Conf. Series: Materials Science and Engineering 862 (2020) 062031. $1-5$ p. https://iopscience.iop.org/article/10.1088/1757899X/862/6/062031

11. K. Abidov, A. Raxmatullaev, O. Zaripov, A.Nuraliev, O.Narzullaeva. Investigation of an electromagnetic two-stroke vibrating activator in oscillatory mode. Journal of Advanced Research in Dynamical and Control Systems. Volume 12. Issue 7 Special Issue, 2020. Pages 2167-2171 